\title{
Biochemical and molecular characterization of orange- and tangerine-colored rice calli
}

\author{
Atsushi Ishihara', Kazuhiko Ohishi ${ }^{2}$, Tetsuya Yamada ${ }^{3}$, Mari Shibata-Hatta ${ }^{4}$, \\ Yuko Arai-Kichise ${ }^{4}$, Satoru Watanabe2 , Hirofumi Yoshikawa ${ }^{2,4}$, Kyo Wakasa ${ }^{2,4, *}$ \\ ${ }^{1}$ Faculty of Agriculture, Tottori University, Tottori, Tottori 680-8553, Japan; ${ }^{2}$ Department of Bioscience, Tokyo University of \\ Agriculture, Tokyo 156-8502, Japan; ${ }^{3}$ Graduate School of Agriculture, Hokkaido University, Sapporo, Hokkaido 060-8589, \\ Japan; ${ }^{4}$ Genome Research Center, NODAI Research Institute, Tokyo University of Agriculture, Tokyo 156-8502, Japan \\ *E-mail: k3wakasa@nodai.ac.jp Tel: +81-3-5477-2769 Fax:+81-3-5477-2377
}

Received May 8, 2015; accepted May 26, 2015 (Edited by Y. Hoshino)

\begin{abstract}
We established an orange (L) and a tangerine (D) colored callus line by repeatedly subculturing rice callus line $(\mathrm{N})$. The L line accumulated lycopene, $\beta$-carotene, and two lycopene isomers, while the $\mathrm{D}$ line accumulated much higher concentration of lycopene as well as a lycopene isomer than the L line. Genomic sequencing revealed the presence of SNP that results in the incomplete lycopene $\beta$-cyclase $(\beta \mathrm{LCY})$ enzyme in the D line. We analyzed expression levels of carotenoid biosynthetic genes by qRT-PCR. Regarding the 2-C-methyl-D-erythritol-4-phosphate pathway genes, expression of the gene encoding geranylgeranyl diphosphate synthase 1 (GGPS1) was enhanced in both $\mathrm{L}$ and D lines as compared to the $\mathrm{N}$ line. Regarding the carotenoid pathway genes, the transcript amounts of the genes encoding phytoene synthase 2, phytoene desaturase, and $\zeta$-carotene desaturase, were larger in the $\mathrm{L}$ line than in the $\mathrm{N}$ line; however, this pattern was not found in the $\mathrm{D}$ line. The expression levels of $\beta L C Y$ and lycopene $\varepsilon$-cyclase $(\varepsilon L C Y)$ gene in the $\mathrm{D}$ line and $\varepsilon L C Y$ in the L line were lower than in the $\mathrm{N}$ line. The transcript amounts of two carotenoid oxygenase genes were decreased both in $\mathrm{L}$ and $\mathrm{D}$ lines. We concluded that increased expression of GGPS1 and reduced expression of $\varepsilon$ LCY, $\beta$ LCY, and carotenoids oxygenase genes might allow an excess accumulation of carotenoids in $\mathrm{L}$ and $\mathrm{D}$ lines, and that the mutation in $\beta$ LCY is responsible for the conspicuous phenotype in the $\mathrm{D}$ line as compared to the L line.
\end{abstract}

Key words: Callus, $\beta$-carotene, carotenoid, lycopene, lycopene $\beta$-cyclase, rice.

Carotenoids are commonly present in most organisms living in aerobic conditions and exposed to sunlight. In plants, carotenoids play an important role in photosynthesis where they work as accessory pigments, harvesting the energy from the sun and transferring it to chlorophyll. These pigments also protect organisms from oxidative damage by reacting with reactive oxygen species generated during photoinhibition processes. In addition, carotenoids accumulate in specific organs such as fruits, roots, and flowers, providing them with characteristic colors. These colors are useful ecological signals, used and recognized by a large range of organisms.

Carotenoids produced by plants are transferred to animals through the food chain and metabolized into functional molecules. Their role as a precursor of vitamin A is particularly important for human health. Vitamin A deficiency can lead to preventable but severe visual impairment and blindness, and significantly increases the chances to contract and develop severe infections, including diarrhea and measles (Underwood and Arthur 1996). Vitamin A and its active metabolite, retinoic acid, are essential for the normal development and function of the hematopoietic and immune systems (Ross et al. 2011). Carotenoids are also important for their own physiological functions. For example, their antioxidant activity may prevent the oxidation of lipids (Di Mascio 1991). Accumulation of carotenoids in the corpus luteum and testicles at a high concentration also suggests its function during animal reproduction (Stahl et al. 1992).

Carotenoid biosynthetic pathway has been well studied in plants. They are tetraterpene molecules derived from the 2-C-methyl-D-erythritol-4-phosphate (MEP) pathway, which generates geranylgeranyl diphosphate (GGPP) (Phillips et al. 2008). Among the enzymes involved in this pathway, 1-deoxy-D-xylulose5-phosphate synthase (DXS), 1-deoxy-D-xylulose-5phosphate reductoisomerase (DXR), and 4-hydroxy-3methylbut-2-enyl diphosphate reductase (HDR) have been suggested to play regulatory roles (Botella-Pavia et al. 2004; Lois et al. 2000). GGPP is synthesized by GGPP synthase (GGPS) from a molecule of dimethylallyl diphosphate and three molecules of isopentenyl diphosphate. GGPP is funneled into the carotenoid 
pathway by phytoene synthase (PSY), which catalyzes the condensation of two molecules of GGPP into phytoene. The conversion of phytoene to lycopene is a four-step desaturation reaction catalyzed by phytoene desaturase (PDS), 15-cis- $\zeta$-carotene isomerase (Z-ISO), $\zeta$-carotene desaturase (ZDS), and carotene isomerase (CRTISO). The cyclization of lycopene ends catalyzed by lycopene $\beta$-cyclase ( $\beta$ LCY) and lycopene $\varepsilon$-cyclase $(\varepsilon$ LCY $)$ forms $\beta$-carotene ( $\beta, \beta$-carotene) or $\alpha$-carotene ( $\beta, \varepsilon$-carotene). Further, xanthophylls are formed by hydroxylation of carotenes by enzymes such as carotene $\beta$-hydroxylases ( $\beta$ OHase), CYP97A, CYP97B and CYP97C. Carotenoids are further converted to apocarotenoids, which include representative phytohormones, abscisic acid (ABA) and strigolactones. In plants, apocarotenoids are biosynthesized by cleavage reactions of carotenoids mediated by dioxygenases such as 9-cis-epoxycarotenoid cleavage dioxygenases (NCEDs) and carotenoid cleavage dioxygenases (CCDs). An ectopic expression of CCDs has generated various cleaved molecules from carotenoids including lycopene and $\beta$-carotene (Auldridge et al. 2006).

Regulation of carotenoid biosynthesis is a complex process. The supply of substrates from the MEP pathway affects carotenoid biosynthesis. The expression of genes encoding DXS and HDR on the MEP pathway correlates with carotenoid accumulation (Carretero-Paulet et al. 2006; Lois et al. 2000), and the overexpression of these genes is known to increase carotenoid content (Carretero-Paulet et al. 2006; Estévez et al. 2001). PSY is generally considered to be an important regulatory point in the control of carotenoid biosynthesis (Cazzonelli and Pogson 2010). The expression of PSY is regulated by $\mathrm{ABA}$, light intensity, salinity, drought, temperature, photoperiod, and developmental cues (Li et al. 2008a, 2008b; Welsch et al. 2008). The expression of CRTISO is also transcriptionally controlled. A chromatin-modifying histone methyltransferase enzyme, SDG8, has been shown to be required for the expression of CRTISO (Cazzonelli et al. 2009, 2010). $\beta$ LCY and $\varepsilon$ LCY activities control the ratio of the most abundant carotenoid, lutein, to other $\beta$-carotenoids. The $\beta$ LCY gene from the eubacterium Erwinia herbicola and daffodil (Narcissus pseudonarcissus L.) flowers were introduced into the tomato (Solanum lycopersicum L.) plastid genome, and lycopene was channeled into the beta-branch, resulting in an increased accumulation of xanthophylls in leaves and predominantly $\beta$-carotene in fruits (Apel and Bock 2009 ). In the absence of $\beta L C Y$, several unusual carotenes have been shown to accumulate in maize (Zea mays L.; Bai et al. 2009). The suppression of $\varepsilon$ LCY expression results in an increase in carotenoid content and the ratio of $\beta$-carotene to lutein in Brassica napus seed (Yu et al. 2008). On the other hand, tuber-specific silencing of the $\varepsilon L C Y$ gene, by introducing an antisense fragment of the gene, increases $\beta$-carotene and zeaxanthin contents in potato tuber (Solanum tuberosum L.; Diretto et al. 2006). The capacity of a metabolic sink is also known to increase carotenoid content in plants. The transformation of $\mathrm{Or}$ gene, promoting the formation of a large membranous chromoplast, leads to an orange color, associated with increased levels of $\beta$-carotene, in cauliflower curds (Brassica oleracea L. var. botrytis) (Li et al. 2001; Lu et al. 2006).

In an effort to provide long-term and sustainable prevention of vitamin A deficiency, scientists have genetically engineered a variety of rice known as the Golden Rice (Ye et al. 2000). The first Golden Rice was generated by introducing three genes: phytoene synthase $(p s y)$ gene from daffodil, carotene desaturase $(c r t I)$ gene from the bacterium Pantoea ananatis, and lycopene $\beta$ cyclase (lcy) from Erwinia uredovora. The genes psy and $l c y$ were expressed under the control of an endospermspecific rice glutelin promoter, while crtI was under the control of a CaMV $35 S$ promoter. The aphIV expressed under the control of CaMV $35 S$ promoter was used as selective marker. However, it was found that the introduction of $l c y$ was not essential to increase the levels of $\beta$-carotene. To increase the level of $\beta$-carotene, the variety Golden Rice 2 was generated by introducing maize psy together with Erwinia uredovora crtI (Paine et al. 2005). The total carotenoid in Golden Rice 2 was up to 23 times higher (maximum $37 \mu \mathrm{g} / \mathrm{g}$ ) than that of the first Golden Rice, with a preferential accumulation of $\beta$-carotene.

Wakasa et al. (1984) established in rice anther culture, the $\mathrm{NR}^{-} 120-2$ callus line $(\mathrm{N})$, derived from pollen and deficient in nitrate reductase. After repeatedly subculturing the calli of this line, we established two callus lines showing orange (L) and tangerine (D) colors. These colors suggested that the pigments accumulating in $\mathrm{L}$ and $\mathrm{D}$ lines might be carotenoids. The calli derived from rice pollen and scutellum typically show white or faint yellow colors; however, to the best of our knowledge, orange rice calli have never been reported. Thus, the biosynthesis of pigments is preferentially suppressed in rice calli generated from these organs. Alternatively, carotenoids may not be accumulated in those calli because of fast flux of carotenoid metabolism. The emergence of calli with orange and tangerine colors may be attributable to the changes in the biosynthesis and/or catabolism of carotenoids through repeated subculturing. Elucidating the underlying mechanism may provide an insight into the regulation of carotenoid metabolism, and may promote the development of new technologies to generate carotenoid-rich rice varieties.

Here, we identified the pigments accumulated in these callus lines and performed genomic analysis by using next generation sequencing (NGS) techniques and gene expression analysis by quantitative RT-PCR, 
to understand the mechanisms associated with the accumulation of these pigments.

\section{Materials and methods}

\section{Callus lines}

Three rice callus lines (Oryza sativa L. cultivar Norin 8 ) were used in this study. Callus line $\mathrm{N}$ is the line 120-2, deficient in nitrate reductase, established by anther culture by Wakasa et al. (1984). The other two callus lines ( $\mathrm{L}$ and $\mathrm{D}$ ) were derived from the callus line $\mathrm{N}$. Line $\mathrm{L}$ is an orange-colored callus that was found after repeatedly subculturing line $\mathrm{N}$ and subsequently isolated. Line D, which shows a tangerine color, was isolated from $\mathrm{L}$ after repeated subcultures. All calli were maintained on AA medium (Müller and Grafe 1978) as previously described (Wakasa et al. 1984).

\section{Protoplast isolation}

Protoplasts were isolated from each callus line (N, L, and D) by treating the calli with Cellulase Onozuka RS (Wako Pure Chemical Industries, Ltd., Osaka, Japan) and Macerozyme R-10 (Yakult Pharmaceutical Co., Ltd., Tokyo, Japan), following a previously described protocol (Wakasa et al. 1984). The suspension obtained was passed through a layer of cell strainer ( $100 \mu \mathrm{m}, \mathrm{BD}$ Bioscience, San Jose, CA, USA) and inspected using an Olympus DP71 digital camera (Olympus, Tokyo, Japan) at $150 \times$.

\section{Extraction of pigments and measurement of absorption spectra}

The calli $(0.1 \mathrm{~g})$ were frozen in liquid $\mathrm{N}_{2}$ and homogenized using a multi-bead shocker (Yasui Kikai, Osaka, Japan) at 1800 rpm for $8 \mathrm{~s}, 28$ days after subculturing. Acetone $(1 \mathrm{ml})$ was added to the homogenate and mixed well. After centrifugation $\left(13,000 \times g, 5 \mathrm{~min}, 4^{\circ} \mathrm{C}\right)$, the supernatant was collected. This procedure was repeated, and a second supernatant was combined with the first supernatant and dried up. The residue obtained was dissolved in $500 \mu \mathrm{l}$ of acetone. This solution was diluted 20 times in acetone, and the absorption spectrum was measured using a Hitachi U-3310 spectrophotometer (Hitachi, Tokyo, Japan).

\section{Chromatographic analyses of pigments}

Callus extracts were analyzed by reversed phase TLC (TLC silica gel $60 \mathrm{RP}-18 \mathrm{~F}_{254} \mathrm{~S}$, Merck, Darmstadt, Germany) and LC-MS. TLC was carried out in an acetone-water $(9: 1, \mathrm{v} / \mathrm{v})$. LC analyses were carried out with a Waters Accquity UPLC coupled with a Quattro Micro mass spectrometer (Waters, Milford, MA, USA). An Accquity UPC BEH C18 1.7- $\mu \mathrm{m}$ column $(2.1 \times 50 \mathrm{~mm}$, Waters $)$ was used for the LC-MS analyses. The solvents used were $0.1 \%$ formic acid in water (solvent A) and acetonitrile-acetone (1:1 ratio $(\mathrm{v} / \mathrm{v})$, solvent $\mathrm{B})$. The analyses were carried out with a $90-99.9 \% \mathrm{~B} /(\mathrm{A}+\mathrm{B})(0-5 \mathrm{~min})$ and $99.9 \% \mathrm{~B} /(\mathrm{A}+\mathrm{B})(5-10 \mathrm{~min})$ gradient. For the LC-PDA analysis, THF-acetonitrile-methanol (7-58-35, v/v) was used as solvent.

\section{DNA extraction}

Genomic DNA was isolated from each callus following the CTAB method (Porebski et al. 1997) with minor modifications. Calli (approx. $30 \mathrm{mg}$ ) were homogenized using a multi-beads shocker in the presence of liquid $\mathrm{N}_{2} 28$ days after subculture. The homogenate was suspended in $400 \mu$ of CTAB buffer $(2 \%$ CTAB, $1.4 \mathrm{M} \mathrm{NaCl}, 20 \mathrm{mM}$ EDTA, $100 \mathrm{mM}$ Tris- $\mathrm{HCl}[\mathrm{pH}$ $8.0]$, and $0.3 \% \beta$-mercaptoethanol). The supernatant $(200 \mu \mathrm{l})$ was subsequently extracted with $200 \mu \mathrm{l}$ of chloroform and centrifuged for $5 \mathrm{~min}$ at $13,000 \times g$. Each upper aqueous phase was transferred into a new tube, and the DNA was precipitated with $500 \mu \mathrm{l}$ of isopropanol and corrected by immediately spinning the mixture at $13,000 \times g$ for $10 \mathrm{~min}$. The DNA pellet was washed with $70 \%$ ethanol, dried, and re-suspended in $50 \mu \mathrm{l}$ of TE solution containing 0.1\% RNaseA (Takara, Ohtsu, Japan).

\section{Genome sequence analysis}

Genomic DNA samples, extracted from calli by using the CTAB method (Porebski et al. 1997), were used for the preparation of sequencing libraries, according to the manufacturer's protocol (Illumina, San Diego, CA, USA). The fragments of the libraries were paired-end sequenced using HiSeq2000 and HiSeq2500 (Illumina). The length of all sequences generated was 100 nucleotides. The NGS reads of these genomes have been submitted to DDBJ (http://www.ddbj.nig.ac.jp/index-e.html) and deposited under the accession number DRA002209.

Sequence mapping and SNP/InDels identification were done according to previously described methods (Arai-Kichise et al. 2014). These data are available at GBrowse, NGRC Orangecolored Rice (http://www.nodai-genome.org/oryza_sativa_ en.html). Detected SNPs and InDels were annotated as gene structures in the Nipponbare genome. When SNPs and InDels were annotated in multiple gene-structures, we selected top three results in ascending order of gene ID.

For direct sequencing of $\beta L C Y, \varepsilon L C Y$, and DXS3 around the SNPs called by the mapping programs, genomic regions were amplified by $\mathrm{PCR}$ using the primer sets ( $\beta$ LCY_f: $5{ }^{\prime}$-CGA CGA GTT CGA CGC CAT GG-3', $\beta$ LCY_r: 5'-GGA CGCGTA TCCCGA GGT-3', $\varepsilon$ LCY_f: 5' GGC TGG CTT TGA TAA TCC AAC TGA A- $3^{\prime}$ and $\varepsilon$ LCY_r: 5' -GCC AAA TTC ATG CAT AAA ATT CTT GCA GTG-3', DXS3_f: 5'-GGA TGC ACG GCG TGG TC-3' and DXS3_r: 5' -CCT CGA GAG GTG TGC CCT T-3') and sequenced on a capillary sequencer with the Sanger method using the commercial sequence service of MACROGEN (Tokyo, Japan).

\section{RNA extraction from callus lines}

Each callus $\left(100 \mathrm{mg}\right.$ ) was frozen in liquid $\mathrm{N}_{2}$ and ground into powder using a mortar and pestle 28 days after subculturing. Total RNA was extracted using TRIzol Reagent (Invitrogen, Carlsbad, CA, USA), according to the manufacturer's instructions. After determination of the RNA concentration, $10 \mu \mathrm{g}$ of RNA was treated with DNase (TURBO DNase, Toyobo, 
Osaka, Japan). Total RNA was purified by an RNeasy MiniElute Cleanup Kit (Qiagen, Venlo, Netherlands).

First-strand cDNA was synthesized from $1 \mu \mathrm{g}$ of purified RNA by reverse transcription using a SuperScript III FirstStrand Synthesis System (Invitrogen) with oligo (dT) primers, according to the manufacturer's instructions. After reverse transcription reaction, RNase $\mathrm{H}$ was added to the reaction mixture and incubated at $37^{\circ} \mathrm{C}$ for $20 \mathrm{~min}$, and the mixture was used as PCR template after 40 times dilution. Quantitative real-time PCR was performed using a StepOnePlus Real-Time PCR System (Applied Biosystems, Foster, CA, USA) with the specific set of primers (Supplemental Table 1). The reaction mixture (final volume $20 \mu \mathrm{l}$ ) contained $2 \mu \mathrm{l}$ of cDNA solution, $0.4 \mu \mathrm{l}$ of forward and reverse primer solutions $(10 \mu \mathrm{M}), 10 \mu \mathrm{l}$ of Fast SYBR Green Master Mix $(\times 2)$ (Applied Biosystems) and $7.2 \mu$ l of DNAase free water. The template $\mathrm{cDNA}$ was denatured at $95^{\circ} \mathrm{C}$ for $20 \mathrm{~s}$, followed by 40 amplification cycles as follows: $95^{\circ} \mathrm{C}$ for $3 \mathrm{~s}, 60^{\circ} \mathrm{C}$ for $30 \mathrm{~s}$, and $95^{\circ} \mathrm{C}$ for $15 \mathrm{~s}$. The reaction mixture was kept at $60^{\circ} \mathrm{C}$ for $1 \mathrm{~min}$ and $95^{\circ} \mathrm{C}$ for $15 \mathrm{~min}$ for drawing melt curve. For the primer sets used, amplification rates were in the range of $80-120 \%$. The relative abundance of transcripts was expressed by the $\Delta \Delta \mathrm{CT}$ method.

\section{Results}

\section{Identification of orange pigments}

We established two callus lines presenting orange (L) and tangerine (D) colors (Figure 1). The L line was isolated from the mutant callus line 120-2 (N), deficient in nitrate reductase (Wakasa et al. 1984) after repeated subculturing. The D line was isolated from a callus that emerged from $\mathrm{L}$ following subsequent repeated subculturing.

To examine the intracellular localization of the orange and tangerine pigments, we prepared protoplasts from $\mathrm{N}, \mathrm{L}$, and $\mathrm{D}$ calli to be observed under a microscope (Figure 2A-2C). The accumulation of pigment was not detected microscopically in protoplasts from line $\mathrm{N}$ (Figure 2A). On the other hand, almost all protoplasts from line $\mathrm{L}$ contained intracellular orange granules and some protoplasts contained red crystalline needlelike structures (Figure 2B). The color of these pigments resembled those observed in red pepper (Capsicum annuum L.) and carrot (Daucus carota L.) protoplasts (Figure 2D, 2E). On the other hand, almost all protoplasts form the $\mathrm{D}$ line contained needle-like red crystals (Figure 2C), which were similar to the crystals observed in tomato protoplasts (Figure 2F). A small portion of the protoplasts from the $\mathrm{D}$ line contained orange granules.

We extracted pigments from N, L, and D calli with acetone and measured the absorption spectra of each extract (Figure 3 ). The extract from $\mathrm{N}$ calli showed a weak absorption, with $\lambda_{\max }$ at $450 \mathrm{~nm}$. The extract from $\mathrm{L}$ showed a level of absorption higher than the $\mathrm{N}$ extract, with $\lambda_{\max }$ at 445,470 , and $524 \mathrm{~nm}$. The extract from D also showed a strong absorption, with $\lambda_{\max }$ at 445,470 and $524 \mathrm{~nm}$, but the shape of the spectrum of the D extract was different from that of the $\mathrm{L}$ extract. In fact, the relative absorbance of peaks at 470 and $524 \mathrm{~nm}$ was larger in D extracts. These spectra were subsequently compared with the spectrum of extracts from tomato fruity flesh and carrot root, whose main pigments are lycopene and $\beta$-carotene, respectively. The spectrum of $\mathrm{D}$ extracts was almost identical to that of tomato extract, suggesting that the main pigment in the $\mathrm{D}$ line was lycopene. In addition, the spectrum of $\mathrm{L}$ extract showed the same $\lambda_{\max }$ as tomato extract, suggesting that the L line also contains large amounts of lycopene as a major pigment. Relatively smaller absorbance at 470 and $524 \mathrm{~nm}$ of L can probably be attributed to the presence of pigments with small $\lambda_{\max }$ values.

Thin layer chromatography (TLC) was used to identify the nature of these pigments (Figure 4). The TLC analysis showed that the main color spots, obtained from the $\mathrm{L}$ and $\mathrm{D}$ extracts, had Rf values of 0.17 identical with that of authentic lycopene (Figure 4A).

The extracts were also analyzed by liquid chromatography coupled with mass spectrometry (LC-MS) and liquid chromatography coupled with photodiode array detection (LC-PDA). The L extract showed four major peaks at 4.15, 4.20, 4.46, and 5.28 min (Figure 4B). The measured retention times of 4.15 and $5.28 \mathrm{~min}$ accurately matched those of purified lycopene and $\beta$-carotene, respectively. ESI-MS and absorption spectra of the 4.15 and 5.28 min peaks (Supplemental Figures $1 \mathrm{C}, 1 \mathrm{~F}, 2 \mathrm{C}$, and $2 \mathrm{~F}$ ) were also identical to those of lycopene and $\beta$-carotene, respectively (Supplemental Figures 1A, 1B, 2A, and 2B). ESI- MS of the 4.20 and 4.46 min peaks (Supplemental Figure 1D, 1E) were closely similar to those of lycopene and $\beta$-carotene, respectively, indicating that they are isomers of these carotenoids. The absorption spectra of the peaks at 4.20 and $4.46 \mathrm{~min}$ showed a $\lambda_{\max }$ at $468 \mathrm{~nm}$ and $440 \mathrm{~nm}$, respectively (Supplemental Figure 2D, 2E).

The D extract showed two major peaks at 4.13 and 4.61 min (Figure 4B). The peak at 4.13 also showed an identical retention time, similar ESI-MS and absorption spectrum with lycopene (Supplemental Figures $1 \mathrm{G}$ and $2 \mathrm{G}$ ). The ESI-MS of the peak at $4.61 \mathrm{~min}$ (Supplemental Figure $1 \mathrm{H}$ ) was very similar to those of lycopene and $\beta$-carotene, suggesting that this compound is also an isomer of these two carotenoids. Its absorption spectrum showed three $\lambda_{\max }$ at 488,457 , and $432 \mathrm{~nm}$ (Supplemental Figure 2H).

The accumulated amounts of lycopene and $\beta$-carotene were determined by HPLC analysis with standard curves generated with authentic compounds. The amounts of unidentified carotenoids eluted at 4.13, 4.20 , and $4.61 \mathrm{~min}$ were estimated on the basis of the 


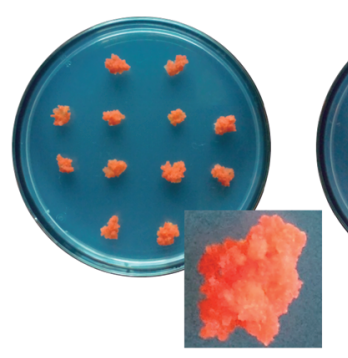

$\mathrm{D}$

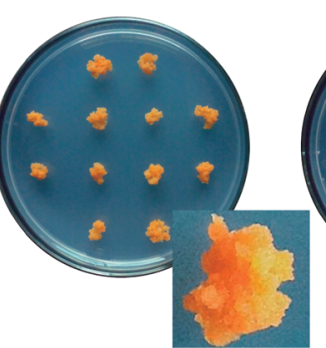

$\mathrm{L}$

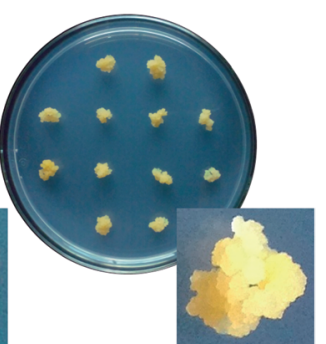

$\mathrm{N}$

Figure 1. Callus lines D (left), L (middle), and N (right). Pictures were taken 28 days after subculturing.
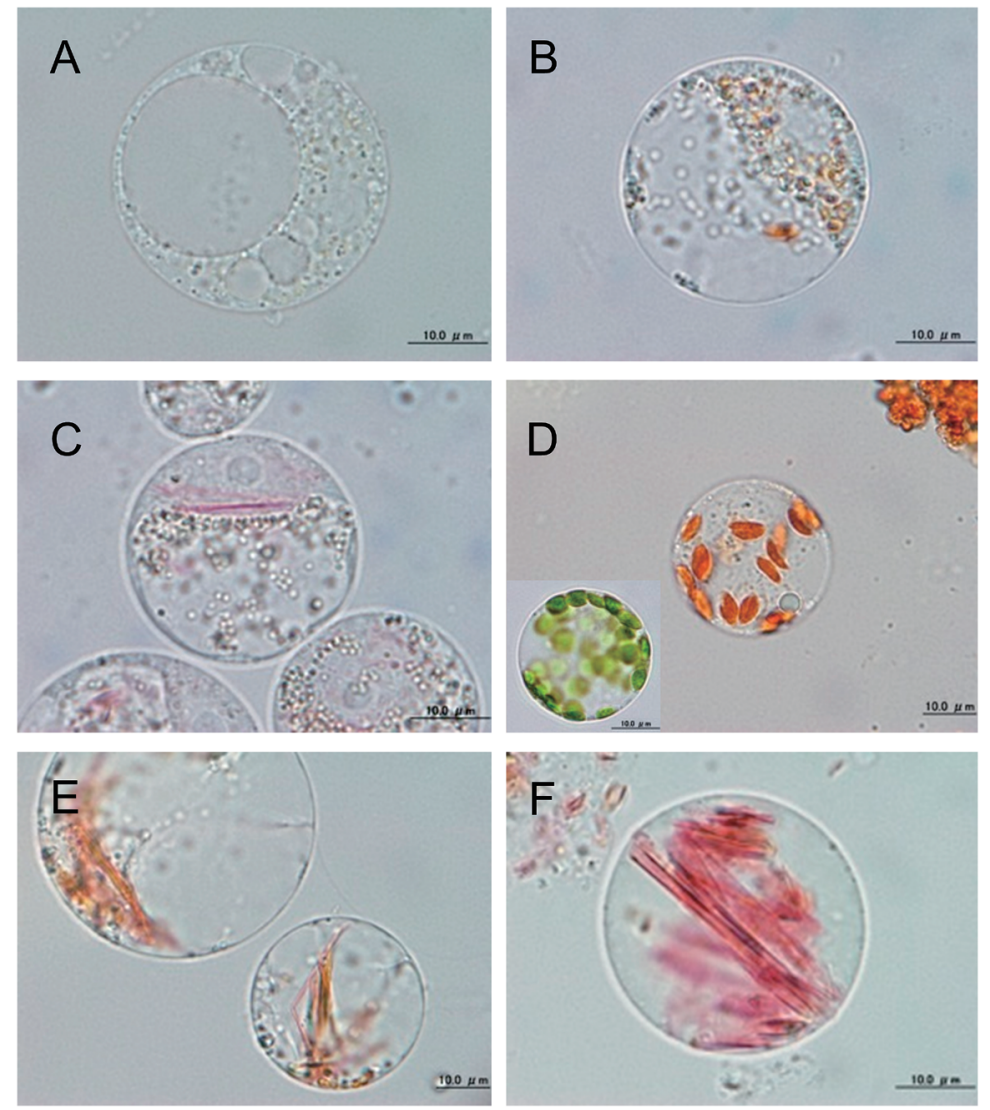

Figure 2. Protoplast isolated from N (A), L (B), and D (C) calli, red and green pepper (D), carrot (E), and tomato (F).

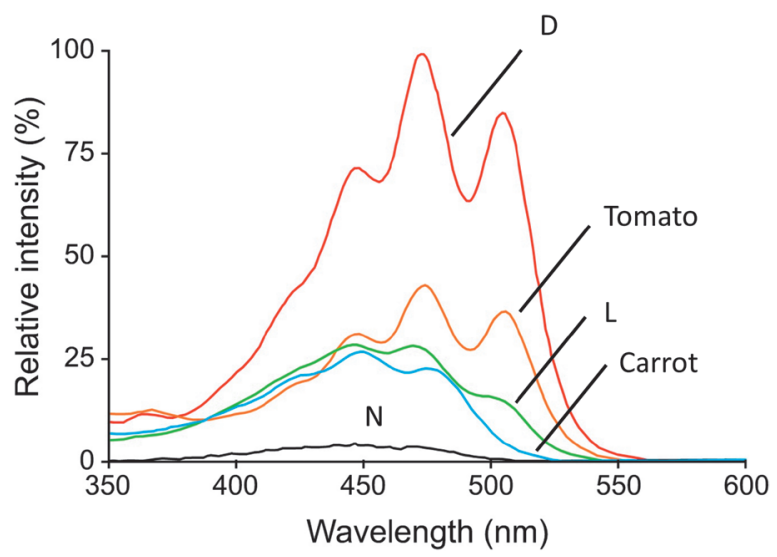

Figure 3. Absorption spectra of extracts from N (black), L (green) and $\mathrm{D}$ (red) callus lines, tomato (orange), and carrot (blue). standard curve for $\beta$-carotene. The $\mathrm{L}$ line accumulated lycopene, $\beta$-carotene, and two unidentified carotenoids, while the D line accumulated mainly lycopene with smaller amounts of another unidentified carotenoid (Table 1). The total amount of carotenoids in D line was approximately ten times larger than that in L line.

\section{Analysis of genome sequences from the $N, L$, and $D$ lines}

Changes in color as a consequence of subculturing can be attributed to carotenoid accumulation in both $\mathrm{L}$ and $\mathrm{D}$ lines. The $\mathrm{D}$ line was derived from the $\mathrm{L}$ line and showed an even more pronounced color phenotype. We analyzed genomic sequences of $\mathrm{N}, \mathrm{L}$, and $\mathrm{D}$ lines using NGS techniques. More than 365 million short-read 
A

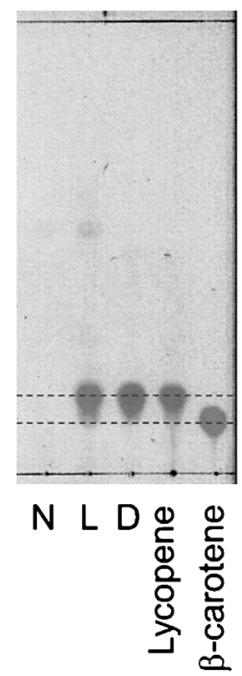

B

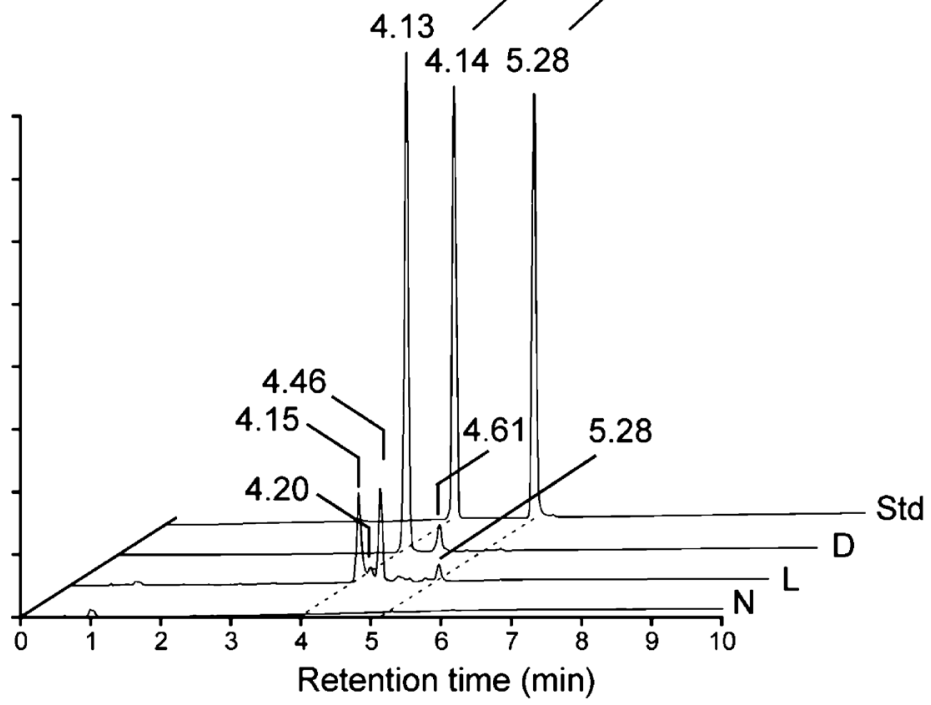

Figure 4. Chromatographic analyses of the pigments isolated from colored callus. The pigments from N, L, and D calli were extracted in acetone and analyzed using TLC (A) with authentic lycopene and $\beta$-carotene. The extracts were also analyzed by UPLC. Standard contains lycopene (retention time: $4.14 \mathrm{~min}$ ) and $\beta$-carotene (retention time: $5.28 \mathrm{~min}$ ). Traces are max plots obtained with detection range $330-600 \mathrm{~nm}$.

Table 1. The accumulation of carotenoids in $\mathrm{N}, \mathrm{L}$, and $\mathrm{D}$ callus lines.

\begin{tabular}{|c|c|c|c|c|c|c|}
\hline Line & Lycopene $^{\mathrm{a}}$ & Rt $4.20^{\mathrm{a}, \mathrm{b}}$ & Rt. $4.46^{\mathrm{a}, \mathrm{b}}$ & Rt. $4.61^{\mathrm{a}, \mathrm{b}}$ & $\beta$-carotene ${ }^{\mathrm{a}}$ & Total $^{\mathrm{a}}$ \\
\hline & $\mu \mathrm{g} / \mathrm{gFW}$ & $\mu \mathrm{g} / \mathrm{gFW}$ & $\mu \mathrm{g} / \mathrm{gFW}$ & $\mu \mathrm{g} / \mathrm{gFW}$ & $\mu \mathrm{g} / \mathrm{gFW}$ & $\mu \mathrm{g} / \mathrm{gFW}$ \\
\hline $\mathrm{N}$ & $0.829 \pm 0.333$ & n.d. ${ }^{c}$ & n.d. ${ }^{c}$ & $0.103 \pm 0.054$ & $0.220 \pm 0.102$ & $1.15 \pm 0.33$ \\
\hline $\mathrm{L}$ & $30.7 \pm 8.80$ & $1.10 \pm 0.36$ & $6.09 \pm 2.27$ & n.d. ${ }^{c}$ & $1.18 \pm 0.20$ & $39.1 \pm 11.0$ \\
\hline D & $406.1 \pm 76.0$ & n.d. ${ }^{c}$ & n.d. ${ }^{c}$ & $20.7 \pm 3.56$ & n.d. ${ }^{c}$ & $426.8 \pm 78.6$ \\
\hline
\end{tabular}

${ }^{a}$ Data represent means \pm SD from three independent replicates. ${ }^{\mathrm{b}}$ Amounts of compounds were calculated by using standard curve generated for $\beta$-carotene. ${ }^{\mathrm{c}}$ n.d.: not detected.

Table 2. Coverage and sequencing depth of mapped reads with reference to the Nipponbare chromosomal genome.

\begin{tabular}{ccccccc}
\hline & \multicolumn{2}{c}{ Mapped reads } & & \multicolumn{3}{c}{ Uniquely mapped reads } \\
\cline { 2 - 3 } & Number of nucleotides (bp) & $\begin{array}{c}\text { Genome coverage with } \\
\text { sequencing depth } \geqq 5(\%)\end{array}$ & Number of nucleotides (bp) & $\begin{array}{c}\text { Genome coverage with } \\
\text { sequencing depth } \geqq 5 \text { (\%) }\end{array}$ & $\begin{array}{c}\text { Average of sequencing } \\
\text { depth (fold) }\end{array}$ \\
\hline $\mathrm{N}$ & $63,185,727,677$ & 98.3 & $31,816,310,259$ & 91.6 & 93.1 \\
$\mathrm{~L}$ & $20,334,420,655$ & 94.1 & $16,360,570,104$ & 87.5 & 50.1 \\
$\mathrm{D}$ & $84,345,207,907$ & 98.4 & $63,828,944,914$ & 92.0 & 185.9 \\
\hline
\end{tabular}

sequences from each callus line were generated. Shortreads from the genome of each line were mapped against the Nipponbare reference genome, and $55.9-75.0 \%$ and $21.5-35.6 \%$ of the total short-reads were successfully assigned to chromosomal and organelle genomes, respectively (Supplemental Table 2). Genomic shortreads were classified into 2 groups: unique or multiple locations. The average sequencing depth of the uniquely mapped reads in each line ranged from $50 \times$ to $185 \times$ across the entire genome, and covered $87.5-92.0 \%$ of the Nipponbare reference genome IRGSP1.0 (Table 2). Thus, all three lines were covered with a sufficient sequencing depth for further genomic analyses (Table 2).

Single-nucleotide polymorphism (SNP) sites were obtained from the three callus lines and annotated using the IRGSP1.0 gene set. All 3 lines presented over 77,000 SNPs and 20,000 insertions and deletions (InDels). From these, more than 5,000 SNPs and approximately 700 InDels were located within a specific gene region (data not shown). These data are available at a GBrowse, NGRC_Orange-colored Rices (http://www.nodaigenome.org/oryza_sativa_en.html).

If carotenoid accumulation is the result of a specific mutation, this mutation would have occurred in the genome of $\mathrm{L}$ and/or $\mathrm{D}$ lines. Although the total number of L-specific SNPs and InDels were 4,314 and 3,906, respectively, only 248 SNPs and 134 InDels were located in coding sequences (Supplemental Table 3). D specific SNPs and InDels were 10,947 and 5,919, respectively, and among them, 590 SNPs and 152 InDels were located 
Table 3. Number of genes containing non-synonymous SNPs and InDels specific to $\mathrm{L}$ and/or $\mathrm{D}$ line.

\begin{tabular}{lcc}
\hline & $\begin{array}{c}\text { Number of genes with } \\
\text { non-synonymous SNP }\end{array}$ & $\begin{array}{c}\text { Number of genes } \\
\text { with InDels }\end{array}$ \\
\hline L specific & 138 & 116 \\
D specific & 242 & 142 \\
LD common & 49 & 21 \\
\hline
\end{tabular}

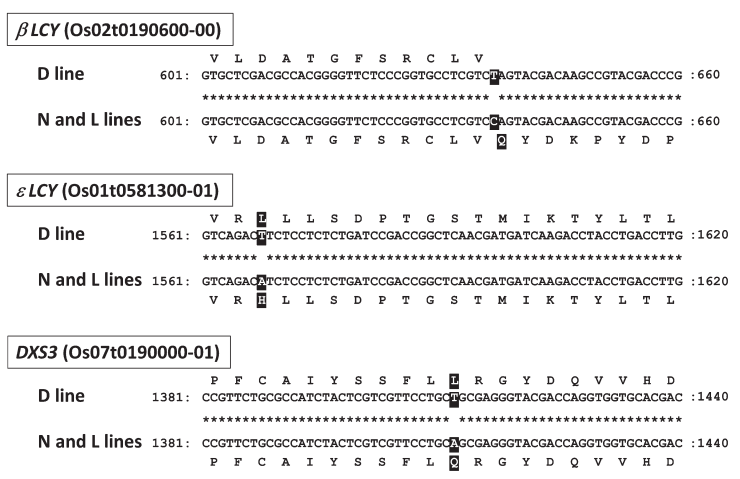

Figure 5. Mutations in $\beta L C Y, \varepsilon L C Y$ and $D X S 3$. The nucleotide sequences around the mutation sites in the ORFs of $\beta L C Y, \varepsilon L C Y$ and DXS3 genes were aligned. Asterisks indicate identical nucleotides, and numbers indicated the positions relative to the translational initiation site in the cDNA sequences. Predicted amino acid sequences were indicated upper and bottom sides of nucleotide sequences. Nucleotides and amino acid residues at the mutation sites were highlighted.

in coding sequences. Among line- specific SNPs, nonsynonymous SNPs were detected in 138 genes in L line and in 242 genes in D line, respectively (Table 3). Only 85 SNPs were found to be common to both $\mathrm{L}$ and $\mathrm{D}$ lines and located in 49 genes. The common InDels between L and $\mathrm{D}$ were as few as 29 and were located in 21 genes.

Focusing on the genes encoding enzymes involved in carotenoid metabolism, one homozygous mutation was detected in the D lines. The SNP resulted in a nonsynonymous substitution in the $\beta L C Y$ gene (locus name: Os02t0190600) of the D line (Figure 5 and Supplemental Figure 3). A direct sequencing analysis confirmed a single base substitution at nucleotide position 637 (relative to the first ATG codon) in the D line. This base substitution resulted in the replacement of glutamine by the stop codon at the putative amino acid position 213. Non-synonymous SNPs, which were heterozygous mutations, were also observed in $\varepsilon$ LCY and DXS3 genes in D and L lines, respectively. The other SNPs of carotenoid-biosynthetic genes detected in both $\mathrm{L}$ and $\mathrm{D}$ lines were silent mutations.

\section{Analysis of expression of carotenoid pathway genes}

DNA polymorphism analysis in $\mathrm{L}$ and $\mathrm{D}$ callus lines revealed the mutations in genes involved in carotenoid biosynthesis. To ensure expression of these genes, and to explore additional, possible causes leading to carotenoid accumulation in L and D lines from a different perspective, we determined the level of transcription of genes involved in both biosynthesis and catabolism of carotenoids by quantitative real-time PCR analyses (Figure 6). We selected genes for the PCR analysis based on the annotation in the RAP-DB, rice annotation project (http://rapdb.dna.affrc.go.jp/).

Regarding the MEP pathway, we analyzed the expression of genes encoding the enzymes DXS, DXR, HDR, and GGPS (Figure 6A). Enhanced expression of these genes would potentially cause the accumulation of lycopene and other carotenoids. DXS1 was transcribed more intensively in $\mathrm{L}$, whereas the amount of DXS3 transcripts was higher in D. The amount of GGPS1 transcripts was higher in both $\mathrm{D}$ and $\mathrm{L}$ lines than in the control (N) line.

Regarding the pathway from GGPP to $\alpha$ - and $\beta$-carotene, we analyzed the amount of transcripts for PSY, PDS, ZDS, CRTISO, $\varepsilon L C Y$, and $\beta L C Y$ (Figure $6 \mathrm{~B})$. The amount of transcripts detected for PSY2, PDS, and $Z D S$ was higher in $\mathrm{L}$, whereas none of the genes upstream of lycopene showed enhanced expression in D. Furthermore, the transcription level of PSY1, PSY3, and $P D S$ was lower in the $\mathrm{D}$ line in comparison with the $\mathrm{N}$ line. The amount of $\varepsilon L C Y$ transcripts was lower in both $\mathrm{D}$ and $\mathrm{L}$ lines, with their respective amounts being $3.4 \%$ and $39 \%$ of that of the $\mathrm{N}$ line. The amount of $\beta L C Y$ transcript in the $\mathrm{D}$ line was $34 \%$ of that of the $\mathrm{N}$ line.

Among the genes involved in the biosynthesis of xanthophylls from carotenes, we analyzed the expression of genes encoding $\beta$-hydroxylase ( $\beta$-OHase) and CYP97s. The amount of $\beta$-OHase 1 transcripts was lower in both $\mathrm{D}$ and $\mathrm{L}$ lines than in the $\mathrm{N}$ line. The amount of $\beta$-OHase2, $\beta$-OHase 3 , and CYP97A transcripts was lower in the $\mathrm{L}$ line, while those of CYP97C were lower in the D line.

We further analyzed the changes of expression of genes annotated as carotenoid dioxygenases based on RAP-DB, because a cleavage reaction of carotenoids mediated by CCDs or NCEDs potentially affect for carotenoid contents. Total five genes, Os02t0704000, Os04t0550600, Os06t0162550, Os08t0371608, and Os12t0640600, were listed as putative carotenoid dioxygenase genes. Os02t0704000, Os04t0550600 and Os12t0640600 were corresponding to CCD4a, CCD7 and CCD1 (Vallabhaneni et al. 2010), respectively, while Os06t0162550 and Os08t0371608 were annotated as genes encoding carotenoid oxygenase domain containing proteins by RAP-DB. Among the analyzed genes, the transcript amounts of Os06t0162550 and Os08t0371608 markedly decreased in $\mathrm{L}$ and $\mathrm{D}$ lines compared with $\mathrm{N}$ line (Figure 7).

\section{Discussion}

We found that $\mathrm{L}$ and $\mathrm{D}$ callus lines accumulated high 
A

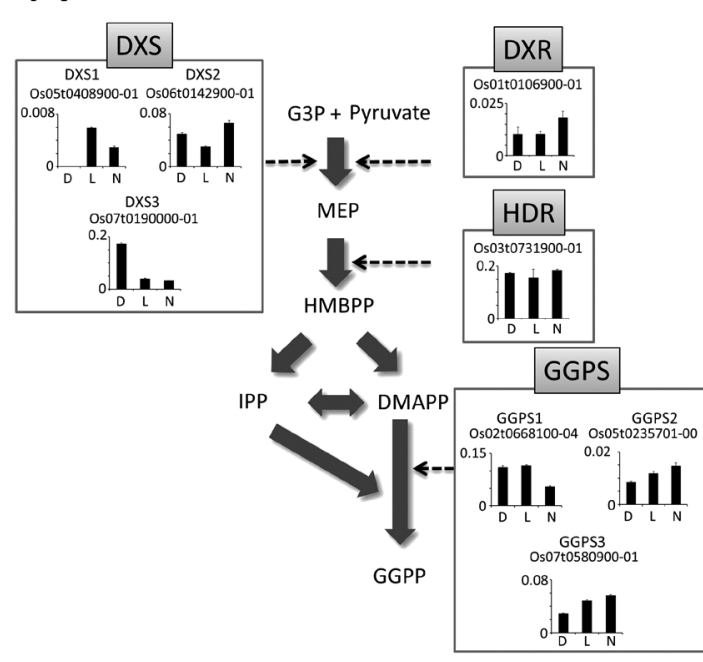

B

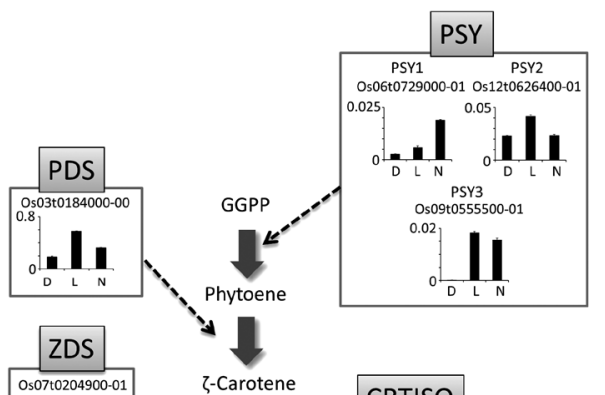

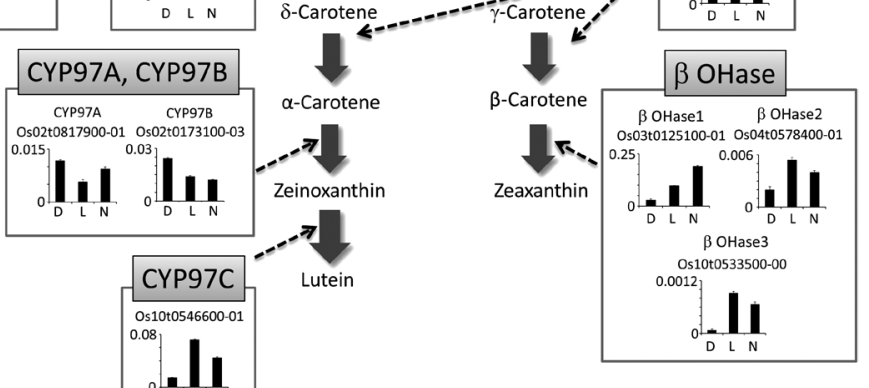

Figure 6. Changes in gene expression in MEP pathway (A) and carotenoid pathway (B). Y-axes of graphs indicate the relative transcript amounts expressed by the $2^{\Delta \mathrm{CT}}$ values determined by qRT-PCR analyses by using actin 1 as internal control. Data represent means \pm SD from three independent replicates.
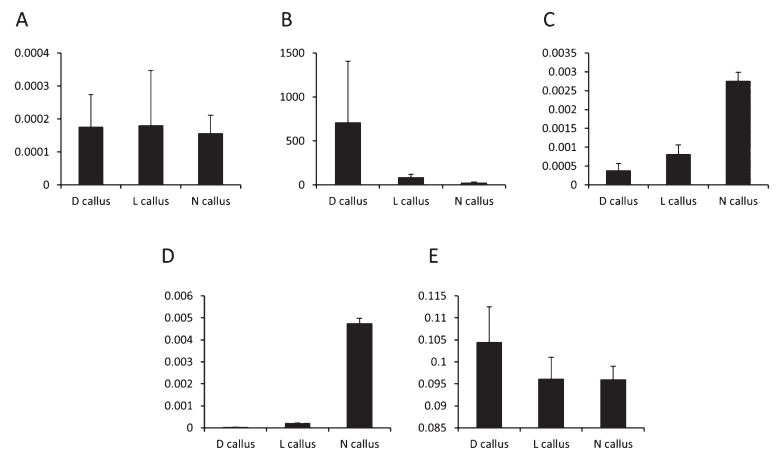

Figure 7. Changes in gene expression in putative carotenoid oxygenases. The changes in the transcript amounts of Os02t0704000 (A), Os04t0550600 (B), Os06t0162550 (C), Os08t0371608 (D), and Os12t0640600 (E) were analyzed by quantitative RT-PCR. Y-axes of graphs indicate the relative transcript amounts expressed by the $2^{\Delta \mathrm{CT}}$ values determined by qRT-PCR analyses by using actin 1 as internal control. Data represent means \pm SD from three independent replicates.

concentrations of carotenoids. Interestingly, L and D callus lines showed the different carotenoid composition; the $\mathrm{L}$ line accumulated lycopene, $\beta$-carotene, and two unidentified carotenoids, while the $\mathrm{D}$ line accumulated lycopene at an extremely high concentration with a minor contribution of an unidentified carotenoid. Microscopic inspection of protoplasts prepared from D line indicated the presence of red needle-like structures that were similar to lycopene crystals in tomato protoplasts. Considering the high concentration of lycopene in D line, the formation of lycopene crystals in the cells is quite reasonable. The L line was isolated through repeated subculturing of the $\mathrm{N}$ line (callus line 120-2), which was a mutant line deficient in nitrate reductase (Wakasa et al. 1984) and did not show enhanced accumulation of carotenoids. The D line was emerged by subsequent repeated subculturing of the $\mathrm{L}$ line. Thus, it is likely that the metabolic changes occurred in $\mathrm{N}$ line led to orange color phenotype, and additional metabolic changes in $\mathrm{L}$ line further led to the more pronounced phenotype in the $\mathrm{D}$ line.

Carotenoids are essential pigments for photosynthetic organisms. In addition, the accumulation of carotenoids in plants gives colors to flowers and fruits, which attract insects and other animals serving as pollinators or seed dispersers. Therefore, carotenoids accumulate in specific organs at specific times in the plant life cycle. Accordingly, carotenoid accumulation is regulated by several different mechanisms. For example, during the ripening of tomatoes, carotenoid accumulation is strongly correlated with the enhanced expression of genes encoding biosynthetic enzymes (Ronen et al. 1999). In citrus species, carotenoid accumulation is 
also correlated with similar changes in gene expression, although the types of carotenoids accumulated in citrus species differ from those in tomato (Kato et al. 2004). The variation in size and number of plastids have also been suggested to affect carotenoid accumulation on the basis of the enhanced accumulation of carotenoids observed in $h p 2$ and $h p 3$ mutants in tomato (Galpaz et al. 2008; Kolotilin et al. 2007). Yellow-colored petals of chrysanthemums accumulate carotenoids, while whitecolored petals lack them because of the presence of carotenoid cleavage dioxygenase (CCD), which degrades carotenoids (Ohmiya et al. 2006). Cassava (Manihot esculenta Crányz) cultivars accumulate carotenoids in their yellow tubers (Welsch et al. 2010). The expression level of genes encoding carotenoid biosynthetic enzymes did not differ from other cultivars. However, in cultivars with yellow tubers, the PSY enzyme, which is considered the rate-limiting enzyme of carotenoid biosynthesis, showed higher enzymatic activity than those in other cultivars because of an amino acid substitution in the enzyme.

Since the color of $\mathrm{L}$ and $\mathrm{D}$ lines emerged after longterm culturing, it is likely that somatic mutations of the regulatory or biosynthetic genes cause the accumulation of carotenoids in these lines. Toward this end, we sequenced the genome of the $\mathrm{D}, \mathrm{L}$, and $\mathrm{N}$ lines by NGS, comparatively analyzed them, and found a mutation in $\beta L C Y$ gene in $\mathrm{D}$ line. This mutation generates a stop codon and results in the formation of a polypeptide with 214 amino acids, which is shorter than the original $\beta$ LCY by 275 amino acid residues. Since this incomplete $\beta$ LCY lacks the protic activation motif (FLEET) that is conserved in plant LCYs (Yu and Beyer 2012), the enzyme is considered inactive. Besides, $50 \%$ of the genomic sequences of $\varepsilon L C Y$ in the D line had a SNP leading to an amino acid substitution. This mutation may also negatively affect the enzymatic function of $\varepsilon L C Y$ because, in general, rarely a mutation improves enzyme function. A similar mutation was found in DXS3 in the L line.

The qRT-PCR revealed the differences in expression level of carotenoid biosynthetic pathway genes among three lines. Several genes in the MEP pathway were upregulated in the L or D lines, but the GGPS1 gene was the only gene whose expression was upregulated in both lines. This upregulation of GGPS1 may support the increased carotenoid biosynthesis in $\mathrm{L}$ and $\mathrm{D}$ lines. Rice genome contains two additional GGPS homologs, GGPS2 and GGPS3. Their expression was not enhanced in $\mathrm{L}$ and $\mathrm{D}$ lines. The estimation of the contribution of each GGPS isozyme to the metabolic flux of the MEP pathway is difficult, but, since the expression level of GGPS1 was much higher than those of other GGPS genes, GGPS1 may greatly contribute to flux of the MEP pathway. In addition, the increased accumulation of DXS3 transcripts in D line may also affect the metabolic flux of the MEP pathway, as DXS is a key step in the MEP pathway. The enhanced expression of DXS has been shown to increase the rate of biosynthesis of carotenoid in tomato (Enfissi et al. 2005) and potato (Morris et al. 2006). The DXS3 transcripts were the most abundant among all DXS transcripts.

Among the genes encoding the carotenoid metabolic pathway enzymes, the amounts of transcripts of the PSY2, PDS, and ZDS genes were increased in the $\mathrm{L}$ line. On the other hand, the transcription of $\varepsilon L C Y$ gene was downregulated both in $\mathrm{L}$ and $\mathrm{D}$ lines, with the degree of decrease in $\mathrm{D}$ line being larger than that in the $\mathrm{L}$ line. In addition, the transcriptional level of $\beta$ $L C Y$ gene decreased in $\mathrm{D}$ line. The level of transcription of $\beta$-OHase1 gene encoding the enzyme that initiates the metabolic pathway for the production of xanthophylls, is also lower both in L and D lines, and it was lower in the $\mathrm{D}$ line than in the $\mathrm{L}$ line. In addition, we detected decreases in the transcript amounts of two genes involved in carotenoid oxidation both in D and L lines with the degree of decrease being larger in the D line. Some of CCD enzymes have an activity to catalyze the cleavage of lycopene and carotene (Auldridge et al. 2006; Bouvier et al. 2005; Giuliano et al. 2003a). However, it remains that other gene products might also be involved in direct catabolism of lycopene and carotenes.

Considering the changes in the gene expression on the whole, in L line, the increased flux of carotenoid biosynthesis by upregulation of PSY2, PDS, and ZDS and suppression of conversion of lycopene into $\alpha$-carotene by downregulation of $\varepsilon L C Y$, as well as the suppression of carotenoid catabolism by carotenoid oxygenases, may have caused the accumulation of lycopene, $\beta$-carotene and other unidentified carotenoids. In $\mathrm{D}$ line, it is highly probable that the mutation of $\beta L C Y$ causes the accumulation of lycopene at an extremely high concentration without the accumulation of $\beta$-carotene. The markedly downregulated expression of $\varepsilon$ LCY in $\mathrm{D}$ line is considered to interfere with funneling the metabolic flux into the other branch in the carotenoid biosynthetic pathway. The strong suppression of the expression of the $\beta$-OHase 3 gene may also be involved in the accumulation of lycopene in the D line. Another possible factor that causes the lycopene accumulation in $\mathrm{L}$ and $\mathrm{D}$ line may be the suppressed conversion of carotenoids to appocarotenoids because the expression of two genes encoding carotenoid oxygenase domain containing proteins were lowered in these lines.

It has been reported that the suppression of $L C Y$ gene expression greatly affects carotenoid composition in plants. The embryos of maize $l c y B$ mutant have been shown to accumulate lycopene and $\delta$-carotene, whereas the endosperm accumulates lycopene, $\delta$-carotene, $\gamma$-carotene, and $\mathcal{\varepsilon}$-carotene (Bai et al. 2009); however, 
these carotenoids are almost absent in the wild type. The suppression of $\varepsilon L C Y$ expression by RNAi techniques in Brassica napus resulted in the increase of carotenoids and in the ratio of $\beta$-carotene to lutein (Yu et al. 2008). In potato, tuber-specific silencing of $\varepsilon L C Y$ gene by introducing an antisense fragment of the gene caused an increase in $\beta$-carotene and zeaxanthin, although not in lutein (Diretto et al. 2006). Differently from these examples, the suppressed expression of both $\varepsilon L C Y$ and $\beta$ $L C Y$ and mutation in $\beta L C Y$ may have caused an extreme increase in lycopene in the $\mathrm{D}$ line.

The enhanced accumulation of PSY2, PDS, and ZDS transcripts was not detected in line $\mathrm{D}$, and their amounts were similar to those in the $\mathrm{N}$ line. In addition, the amount of PSY1 and PSY 3 transcripts in the D line were much smaller than that in the $\mathrm{N}$ line. Maize $l c y B$ mutant accumulates carotenoids, including lycopene, at high concentrations in embryos and endosperm, and it shows a suppressed accumulation of Psy 1 transcript, suggesting a feedback regulation mechanism of carotenoid biosynthesis by accumulation of downstream carotenoids (Bai et al. 2009). Lycopene or unidentified carotenoids accumulated at extremely high concentrations in line D, possibly leading to a feedback mechanism responsible for the observed lack of enhanced expression of lycopene biosynthetic genes.

In the present study, we detected a mutation in $\beta$ $L C Y$ gene in D line, leading to formation of a truncated polypeptide, when we extracted the genes with the mutation in $90 \%$ of read depth. We performed direct sequencing $\beta L C Y$ gene, and confirmed that the mutation is homozygous and specific to D line. All genes of the $\mathrm{N}$ line are theoretically homozygous, because it was derived from pollen through anther culture. Thus, the heterozygosity generated by a somaclonal mutation in $\beta L C Y$ is considered to be lost during repeated subculturing. Loss of heterozygosity $(\mathrm{LOH})$ has been well characterized in human cancers (Lasko et al. 1991). Tumor cells exhibit various forms of genetic instability including chromosomal deletion, mitotic nondisjunction, and recombination between homologous chromosomes, and these genetic instability causes $\mathrm{LOH}$. Since enhanced genetic instability was also indicated in plant tissue cultures (Phillips et al. 1994), the LOH of the mutated $\beta L C Y$ allele in the $\mathrm{D}$ line during subculturing is not surprising.

Here, we report the establishment of two callus lines that accumulated carotenoids at high concentrations. The qRT-PCR analysis of the lines suggested that the activation of carotenoid biosynthesis and the suppression of the conversion of accumulated carotenoids are one of the causes of carotenoid accumulation. The changes in expression of multiple genes in the carotenoid biosynthetic pathway suggest the mutation of transcriptional factor(s) regulating the gene expression, but it remains unsolved. In addition, we could not exclude the possibility of any epigenetic changes such as DNA methylation and chromatin modifications (Lopez et al. 2010; Miguel and Marum 2011). Elucidating the regulatory mechanisms underlying the changes in the expression of the carotenoid biosynthetic pathway genes should help promoting the expansion of new technologies for the development of carotenoidbiofortified rice cultivars by manipulating mutated rice genes.

\section{Acknowledgements}

This study was supported by a MEXT-Supported Program for the Strategic Research Foundation at Private Universities (S0801025 and S1311017).

\section{References}

Apel W, Bock R (2009) Enhancement of carotenoid biosynthesis in transplastomic tomatoes by induced lycopene-to-provitamin A conversion. Plant Physiol 151: 59-66

Arai-Kichise Y, Shiwa Y, Ebana K, Shibata-Hatta M, Yoshikawa H, Yano M, Wakasa K (2014) Genome-wide DNA polymorphisms in seven rice cultivars of temperate and tropical japonica groups. PLoS ONE 9: e86312

Auldridge ME, McCarty DR, Klee HJ (2006) Plant carotenoid cleavage oxygenases and their apocarotenoid products. Curr Opin Plant Biol 9: 315-321

Bai L, Kim E-H, DellaPenna D, Brutnell TP (2009) Novel lycopene epsilon cyclase activities in maize revealed through perturbation of carotenoid biosynthesis. Plant J 59: 588-599

Botella-Pavía P, Besumbes Ó, Phillips MA, Carretero-Paulet L, Boronat A, Rodríguez-Concepción M (2004) Regulation of carotenoid biosynthesis in plants: evidence for a key role of hydroxymethybutenyl diphosphate reductase in controlling the supply of plastidial isoprenoid precursors. Plant J 40: 188-199

Bouvier F, Isner JC, Dogbo O, Camara B (2005) Oxidative tailoring of carotenoids: a prospect towards novel functions in plants. Trends Plant Sci 10: 187-194

Carretero-Paulet L, Cairo A, Botella-Pavía P, Besumbes O, Campos N, Boronat A, Rodríguez-Concepción M (2006) Enhanced flux through the methylerythritol 4-phosphate pathway in Arabidopsis plants overexpressing deoxyxylulose 5-phosphate reductoisomerase. Plant Mol Biol 62: 683-695

Cazzonelli CI, Cuttriss AJ, Cossetto SB, Pye W, Crisp P, Whelan J, Finnegan EJ, Turnbull C, Pogson BJ (2009) Regulation of carotenoid composition and shoot branching in Arabidopsis by a chromatin modifying histone methyltransferase, SDG8. Plant Cell 21: 39-53

Cazzonelli CI, Pogson BJ (2010) Source to sink: regulation of carotenoid biosynthesis in plants. Trends Plant Sci 15: 266-274

Cazzonelli CI, Roberts AC, Carmody ME, Pogson BJ (2010) Transcriptional control of SET DOMAIN GROUP8 and CAROTENOID ISOMERASE during Arabidopsis development. Mol Plant 3: 174-191

Di Mascio P, Murphy ME, Sies H (1991) Antioxidant defense systems: the role of carotenoids, tocopherols, and thiols. Am J Clin Nutr 97(Suppl): 194S-200S

Diretto G, Tavazza R, Welsch R, Pizzichini D, Mourgues F, Papacchioli V, Beyer P, Giuliano G (2006) Metabolic engineering 
of potato tuber carotenoids through tuber-specific silencing of lycopene epsilon cyclase. BMC Plant Biol 6: 13

Estévez JM, Cantero A, Reind A, Reichler S, León P (2001) 1-Deoxy-D-xylulose-5-phosphate synthase, a limiting enzyme for plastidic isoprenoid biosynthesis in plants. J Biol Chem 276: 22901-22909

Enfissi EMA, Fraser PD, Lois LM, Boronat A, Schuch W, Bramley PM (2005) Metabolic engineering of the mevalonate and nonmevalonate isopentenyl diphosphate-forming pathways for the production of health-promoting isoprenoids in tomato. Plant Biotechnol J 3: 17-27

Galpaz N, Wang Q, Menda N, Zamir D, Hirschberg J (2008) Abscisic acid deficiency in the tomato mutant high-pigment 3 leading to increased plastid number and higher fruit lycopene content. Plant J 53: 717-730

Giuliano G, Al-Babili S, von Lintig J (2003) Carotenoid oxygenases: cleave it or leave it. Trends Plant Sci 8: 145-148

Kato M, Ikoma Y, Matsumoto H, Sugiura M, Hyodo H, Yano M (2004) Accumulation of carotenoids and expression of carotenoid biosynthetic genes during maturation in citrus fruit. Plant Physiol 134: 824-837

Kolotilin I, Koltai H, Tadmor Y, Bar-Or C, Reuveni M, Meir A, Nahon S, Shlomo H, Chen L, Levin I (2007) Transcriptional profiling of high pigment-2 $2^{d g}$ tomato mutant links early fruit plastid biogenesis with its overproduction of phytonutrients. Plant Physiol 145: 389-401

Lasko D, Cavenee W, Nordenskjöld M (1991) Loss of constitutional heterozygosity in human cancer. Annu Rev Genet 25: 281-314

Li F, Vallabhaneni R, Wurtzel ET (2008a) PSY3, a new member of the phytoene synthase gene family conserved in the Poaceae and regulator of abiotic stress-induced root cartenogenesis. Plant Physiol 146: 1333-1345

Li F, Vallabhaneni R, Yu J, Rocheford T, Wurtzel ET (2008b) The maize phytoene synthase gene family: overlapping roles for carotenogenesis in endosperm, photomorophogenesis, and thermal stress tolerance. Plant Physiol 147: 1334-1346

Li L, Paolillo DJ, Parthasarathy MV, Dimuzio EM, Garvin DF (2001) A novel gene mutation that confers abnormal patterns of beta-carotene accumulation in cauliflower (Brassica oleracea var. botrytis). Plant J 26: 59-67

Lois LM, Rodríguez-Concepción M, Gallego F, Campos N, Boronat A (2000) Carotenoid biosynthesis during tomato fruit development: regulatory role of 1-deoxy-D-xylulose 5-phosphate synthase. Plant J 22: 503-513

Lopez CMR, Wetten AC, Wilkinson MJ (2010) Progressive erosion of genetic and epigenetic variation in callus-derived cocoa (Theobroma cacao) plants. New Phytol 186: 856-868

Lu S, Eck Van J, Zhou X, Lopez AB, O'Halloran DM, Cosman KM, Conlin BJ, Paolillo DJ, Garvin DF, Vrebalov J, et al. (2006) The cauliflower Or gene encodes a DnaJ cysteine-rich domaincontaining protein that mediates high levels of beta-carotene accumulation. Plant Cell 18: 3594-3605

Miguel C, Marum L (2011) An epigenetic view of plant cells cultured in vitro: somaclonal variation and beyond. J Exp Bot 62: 3713-3725

Morris WL, Ducreux LJ, Hedden P, Millam S, Taylor MA (2006) Overexpression of a bacterial 1-deoxy-D-xylulose 5-phosphate synthase gene in potato tubers perturbs the isoprenoid metabolic network: implications for the control of the tuber life cycle. J Exp Bot 57: 3007-3018

Müller AJ, Grafe R (1978) Isolation and characterization of cell lines of Nicotiana tubacum laking nitrate reductase. Mol Gen Genet 177: 145-153

Ohmiya A, Kishimoto S, Aida R, Yoshioka S, Sumitomo K (2006) Carotenoid cleavage dioxygenase (CmCCD4a) contributes to white color formation in chrysanthemum petals. Plant Physiol 142: 1193-1201

Paine JA, Shipton CA, Chaggar S, Howells RM, Kennedy MJ, Vernon G, Wright SY, Hinchliffe E, Adams JL, Silverstone AL, et al. (2005) Improving the nutritional valueof Golden Rice through increased pro-vitamin A content. Nat Biotechnol 23: 482-487

Phillips MA, León P, Boronat A, Rodríguez-Concepción M (2008) The plastidial MEP pathway: unified nomenclature and resources. Trends Plant Sci 13: 619-623

Phillips RL, Kaepplert SM, Olhoft P (1994) Genetic instability of plant tissue cultures: Breakdown of normal controls. Proc Natl Acad Sci USA 91: 5222-5226

Porebski S, Bailey LG, Baum BR (1997) Modification of a CTAB DNA extraction protocol for plants containing high polysaccharide and polyphenol components. Plant Mol Biol Rep 15: 8-15

Ronen G, Cohen M, Zamir D, Hirschberg J (1999) Regulation of carotenoid biosynthesis during tomato fruit development: expression of the gene for lycopene epsilon-cyclase is downregulated during ripening and is elevated in the mutant Delta. Plant J 17: 341-351

Ross AC, Chen Q, Ma Y (2011) Vitamin A and retinoic acid in the regulation of B-cell development and antibody production. Vitam Horm 86: 103-126

Stahl W, Schwarz W, Sundquist AR, Sies H (1992) cis-trans isomers of lycopene and $\beta$-carotene in human serum and tissues. Arch Biochem Biophys 294: 173-177

Underwood BA, Arthur P (1996) The contribution of vitamin A to public health. FASEB J 10: 1040-1048

Vallabhaneni R, Bradbury LM, Wurtzel ET (2010) The carotenoid dioxygenase gene family in maize, sorghum, and rice. Arch Biochem Biophys 504: 104-111

Wakasa K, Kobayashi M, Kamada H (1984) Colony formation from protoplasts of nitrate reductase-deficient rice cell lines. J Plant Physiol 117: 223-231

Welsch R, Arango J, Bär C, Salazar B, Al-Babili S, Beltrán J, Chavarriaga P, Ceballos H, Tohme J, Beyer P (2010) Provitamin A accumulation in cassava (Manihot esculenta) roots driven by a single nucleotide polymorphism in a phytoene synthase gene. Plant Cell 22: 3348-3356

Welsch R, Wust F, Bar C, Al-Babili S, Beyer P (2008) A third phytoene synthase is devoted to abiotic stress-induced ABA formation in rice and defines functional diversficationof PYSs. Plant Physiol 147: 367-380

Ye X, Al-Babili S, Klöti A, Zhang J, Lucca P, Beyer P, Potrykus I (2000) Engineering the provitamin A (beta-carotene) biosynthetic pathway into (carotenoid-free) rice endosperm. Science 287: 303-305

Yu B, Lydiate DJ, Young LW, Schäfer UA, Hannoufa A (2008) Enhancing the carotenoid content of Brassica napus seeds by downregulating lycopene epsilon cyclase. Transgenic Res 17: 573-585

Yu Q, Beyer P (2012) Reaction specificities of the $\varepsilon$-ionone-forming lycopene cyclase from rice (Oryza sativa) elucidated in vitro. FEBS Lett 586: 3415-3420 